\title{
One-stop haematuria clinic: First experience in South Africa
}

\author{
S Sinha, ${ }^{1}$ MBBS, MS, FRCS (Glasg), FC Urol (SA); S Z Jaumdally, ${ }^{2} \mathrm{PhD}$; J John, ${ }^{1,3}$ MB ChB; G Pinto, ${ }^{1}$ MD, FC Urol (SA); S Sinha, ${ }^{1}$ MB ChB; \\ J Lazarus, ${ }^{1}$ MB ChB, MMed (Urol), FC Urol (SA) \\ ${ }^{1}$ Division of Urology, Department of Surgery, Faculty of Health Sciences, University of Cape Town and Groote Schuur Hospital, Cape Town, South Africa \\ ${ }^{2}$ Division of Immunology, Institute of Infectious Diseases and Molecular Medicine, University of Cape Town, South Africa \\ ${ }^{3}$ Department of Urology, Frere Hospital and Faculty of Health Sciences, Walter Sisulu University, East London, South Africa
}

Corresponding author: S Sinha (sinhasj@hotmail.com)

\begin{abstract}
Background. Haematuria is the most common symptom of urological cancers, specifically bladder cancer, and timely diagnosis can prevent disease from progressing to a more advanced or incurable stage. One-stop haematuria clinics (OSHCs) have become commonplace in urological services in developed countries during the past three decades.

Objectives. To assess the efficacy of this specialised clinic, aimed at providing an investigative service for patients with haematuria, in decreasing morbidity and mortality by earlier diagnosis of urological malignancy. We also report on the outcomes of this study.

Methods. A total of 275 patients who attended the weekly OSHC at Groote Schuur Hospital, Cape Town, South Africa (SA), between January 2012 and October 2015 were retrospectively included in the study (out of 477 folders reviewed). Only patients with visible haematuria (275/477) were included, and characteristics such as gender, age, self-identified ethnicity, and outcomes following OSHC attendance (diagnoses and stage/grade/type of cancers) were recorded.

Results. While the majority of cases were classified as indeterminate following investigation, one-fifth (55/275) of the patients were diagnosed with urological neoplasms, mainly bladder cancer $(87.2 \%, n=48)$. The 50 - 69 -year age group was the most common window for diagnosis of a neoplasm. Forty-six patients (46/55) with urothelial cancers were diagnosed at a relatively early stage and were therefore offered curative management; 5 patients presented with late-stage disease and risked poor outcomes after management. The remaining 4 identified cases were adenocarcinomas and squamous cell carcinomas. Most patients presented with high-grade cancers (43.2\%). A small subset of patients were diagnosed with renal cell carcinoma (11.6\%) and upper-tract transitional cell carcinoma (1.6\%).

Conclusions. This audit revealed that an OSHC can streamline diagnosis of urological malignancies in the SA setting, and highlights the fact that the patients most at risk for developing malignant conditions were the ones frequently diagnosed at a later stage and hence potentially facing a poorer prognosis. These findings support the setting up of such clinics in other SA hospitals to improve ease of early access to the urological service.
\end{abstract}

S Afr Med J 2019;109(11):850-853. https://doi.org/10.7196/SAMJ.2019.v109i11.13827

Haematuria, whether macroscopic (visible) or microscopic (nonvisible), is one of the most common reasons for referral to a urologist. ${ }^{[1]}$ It is estimated to account for one-fifth of all urological visits and for a significant proportion of all urological hospitalisations. ${ }^{[2]}$ This common symptom has a multitude of differential diagnoses, broadly classified into urological and nephrological causes.

Urological causes range from benign conditions such as idiopathic microscopic haematuria to advanced genitourinary malignancies. Evidence shows that as many as $24 \%$ of patients with haematuria are diagnosed with bladder, upper urinary tract and renal tumours, and up to $53 \%$ of those initially considered to have benign conditions are found to have genitourinary malignancies. ${ }^{[2-4]}$ It is therefore vital that evaluation of haematuria is initiated as soon as possible to avoid the morbidity and mortality associated with a delayed diagnosis.

Bladder cancer ranks in the top 10 most prevalent histologically diagnosed cancers in South Africa (SA). It carries a varying estimated lifetime risk in the population, with factors such as gender and race contributing significantly to the risk of developing the malignancy. In SA, individuals self-identifying as white and coloured males had the highest risk profile, with an estimated life-risk of 1:60 and 1:76, respectively, of developing the disease (CANSA Fact Sheet, 2014 $4^{[5]}$ ). Smoking remains the behavioural factor most strongly associated with the development of urothelial cancers. ${ }^{[6]}$

It is known that bladder transitional cell carcinoma (TCC) can progress to muscle invasion in a relatively short period of time, and it is therefore of critical importance that screening and diagnostic services are made as accessible as possible to communities at risk to capture urothelial malignancies early in their development. ${ }^{[7]}$ In settings where such facilities have been established for a while, the rate of diagnosis of TCC is higher than in places without them, enabling early initiation of treatment and therefore markedly improving patient prognosis. ${ }^{[8,9]}$

\section{Objectives}

The one-stop haematuria clinic (OSHC) at Groote Schuur Hospital (GSH) in Cape Town, SA, was established in 2008 with the goal of ensuring rapid and complete assessment by a consultant urologist at a single hospital visit. The objectives of this study were to provide an overall audit of the feasibility and practicality of this approach and to report on the collected outcomes of patients seen at the OSHC over a 3.5 -year period.

\section{Methods}

\section{Patient selection}

A retrospective assessment of folders for patients reporting to the weekly GSH OSHC for the period January 2012 - October 2015 was conducted following ethical approval from the Faculty of Health Sciences Human Research Ethics Committee at the University of Cape Town (ref. no. 868/2015). The clinic is open for 3 hours every Monday afternoon, from an outpatient setting, and the consultant 
sees between three and five patients a week. Patients are referred to the clinic by general practitioners and primary and secondary public hospitals, and there is also interdepartmental referral within GSH. Data collection was done by SS (first author), JJ, GP, SS and JL. The presence of visible haematuria at the time of the visit qualified a patient for inclusion into the study, while patients with microscopic haematuria and those reporting for follow-up visits were excluded.

\section{Procedure}

Every patient visiting the clinic was seen by a consultant urologist, and the consultation started with a focused history. Emphasis was placed on identifying risk factors for urothelial malignancies, including but not limited to age $>50$ years, a family history of urological malignancies, a history of smoking, and exposure to occupational urothelial carcinogens. After a focused physical examination, urinalysis was performed to exclude any ongoing urinary tract infection and to identify proteinuria, which would suggest a possible nephrological cause of haematuria. A kidney-ureter-bladder (KUB) ultrasound scan was then performed. An explanation of planned investigations was given and consent was obtained from the patient, followed by flexible cystoscopy under local anaesthesia.

If no significant pathology and no identifiable risk factors were observed, patients were reassessed within a year of their initial visit at the GSH urology outpatient clinic. Those classified as negative for haematuria at this follow-up visit were advised to return in the event of any recurrence of symptoms. Patients with a suspected nephrological cause of macroscopic haematuria were referred to the renal unit. Those requiring further investigations (urine cytology, computed tomography/magnetic resonance imaging, urography) were followed up in the urology outpatient clinic. Finally, patients with identifiable pathology and needing further intervention (transrectal ultrasound biopsy of the prostate, transurethral resection of bladder tumour, radical nephrectomy or stone surgery) were counselled and placed on the waiting list.

\section{Statistical analysis}

Explorative univariate statistical analysis of the extracted data was done. Statistical inferences on binary sets of data were performed using Fisher's exact test. Statistical analyses were performed using GraphPad Prism version 5.0 for Windows (GraphPad
Software, USA). All tests were two-tailed and $p$-values $<0.05$ were considered significant.

\section{Results}

A total of 477 patients referred to the OHSC at GSH were reviewed for inclusion into the study. Of these, 26 were classified as having microscopic haematuria, and a further 174 were identified as follow-up patients and were excluded. Two further participants with missing records for essential end-points for the study were also excluded. A final set of 275 patients were retrospectively included into the study, with a median age of 60 years (interquartile range 49 - 69). Ages were similar for the 198 men $(72.0 \%)$ and 77 women $(28.0 \%)$; most patients were aged $>50$ years $(73.1 \%)$, with the $60-69$-year age group being the most populated category (29.5\%) (Fig. 1A). A significant majority $(75.6 \%)$ of participants in the study selfidentified as coloured when asked about their ethnicity, and more than half of

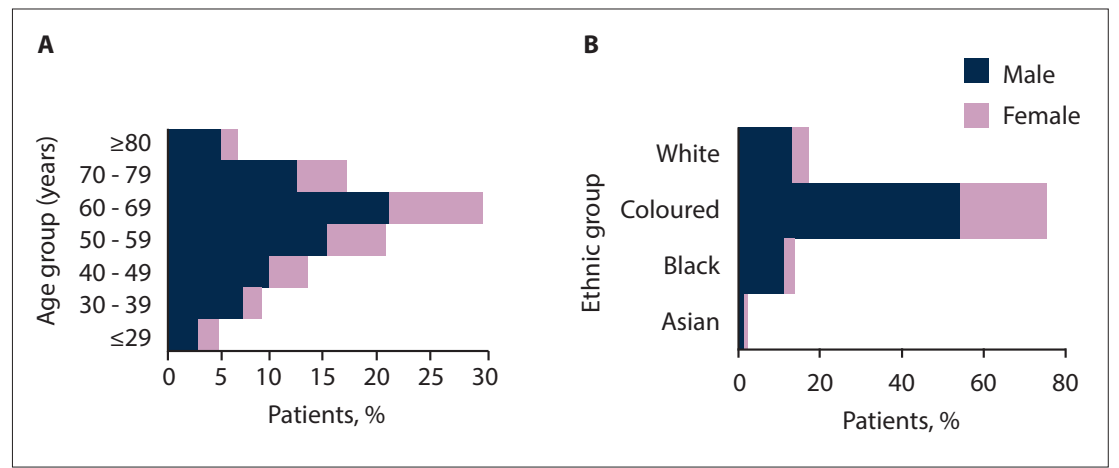

Fig. 1. Patient age distribution (A) and self-identified ethnic distribution (B), by gender.

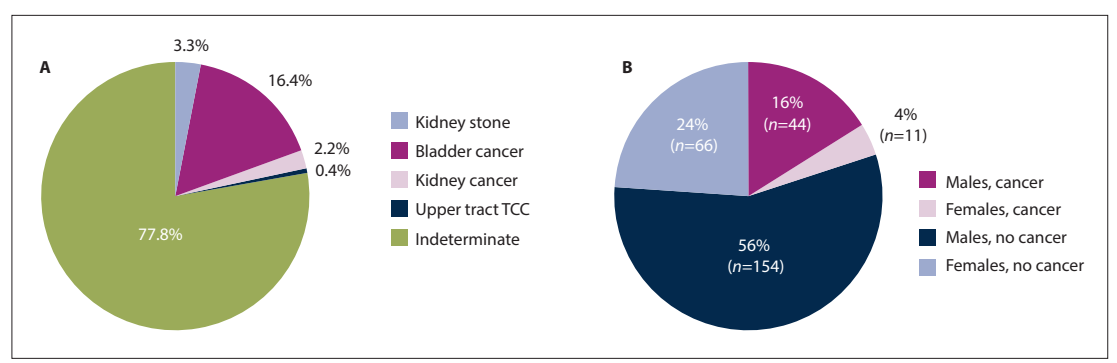

Fig. 2. Percentage breakdown of diagnoses for included cases $(A)$ and incidence of cancers in included cases (B). (TCC = transitional cell carcinoma.)

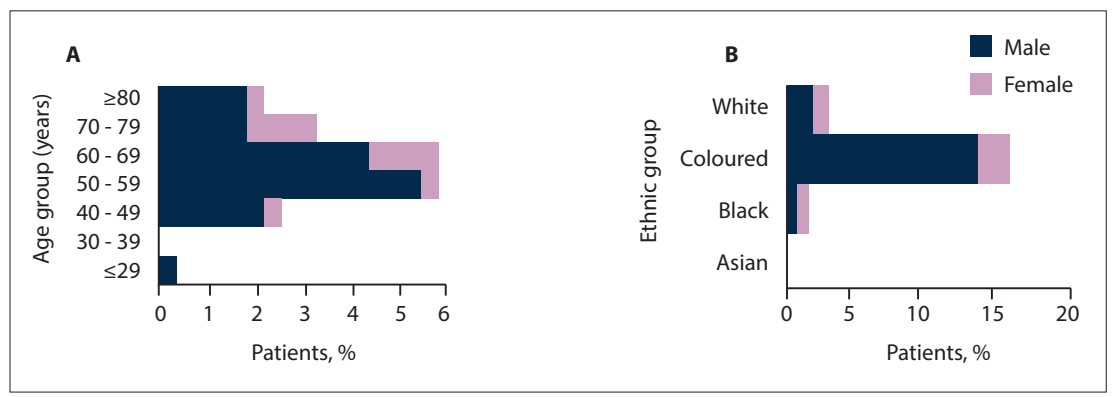

Fig. 3. Distribution of cancers across age (A) and self-identified ethnicity, by gender (B). 
cancers for the various age groups, with $58.2 \%$ of cancers detected in patients in the 50 -69-year group and coloured males making up the majority of detected cancer cases (67.5\%) (Fig. 3B).

\section{Staging and grading}

Of the 55 cancer cases, 51 were classified as TCC and 2 each as adenocarcinoma and squamous cell carcinoma (Fig. 4A). Staging of the TCC cases revealed that 46 of them classified as localised (carcinoma in situ (CIS), Ta, T1 and T2) and 5 as locally advanced cancers (T3 and T4) (Fig. 4B). Finally, TCC high grade (HG) represented the most commonly identified cancer in this study (43.2\%, Fig. 4C). No concurrent CIS was identified. No relevant pattern of interest was observed when looking at grading and staging of cases across the ethnic groups (data not shown).

\section{Discussion}

OSHCs have been in existence for more than three decades globally. ${ }^{[10]}$ With the intention of moving away from the traditional format where diagnostic tests are typically ordered at the first visit to the urologist and a followup appointment is required to assess the results, the aim of an OSHC is to provide a streamlined and rapid investigative service for all patients presenting with haematuria in an effort to decrease morbidity and mortality by earlier diagnosis of urological malignancy. Evidence shows that early diagnosis considerably improves the 1-year survival rate for bladder and renal malignancies, and that this can be as high as $92-97 \%$. However, if patients are diagnosed late, the rate decreases significantly to 25 - 34\%. ${ }^{[1]}$ It is therefore important to make the diagnosis as soon as possible and initiate treatment without delay. This is not always achievable in an overburdened public healthcare system, where delays in initiating treatment represent the main factor contributing to cancer morbidity and mortality. The present study excluded patients with invisible haematuria and those who were attending for follow-up visits. A recent prospective analysis confirmed that patients with dipstick haematuria and negative investigations are extremely unlikely to have significant urological disease. ${ }^{[4]}$ It must be stated that although priority is given at our OSHC to patients with visible haematuria, patients with microscopic haematuria are still worked up according to international guidelines in the urology outpatient department.

In the majority of patients (77.8\%), the cause of the macroscopic haematuria was indeterminate and transient, and resolved without treatment. Where a cause was identified, malignancies of the upper genitourinary tract, kidney and bladder were responsible for most of the macroscopic haematuria. In line with previous reports, ${ }^{[4,12,13]}$ a relatively small fraction $(3.3 \%)$ of patients in our study were diagnosed with kidney stones. Other common conditions such as urinary tract infection and benign prostate hyperplasia have not been described individually here, as we believe that they accounted for the majority of the indeterminate group in this study. Bladder cancer alone accounted for $16.4 \%$ of macroscopic haematuria. According to incidence reports from the 2014 South African National Cancer Registry, ${ }^{[5]}$ bladder cancer remains among the top 10 cancers diagnosed in South Africans. The ethnic and gender links to bladder cancer incidence found in the present study cannot be generalised to a nationwide context, mainly because of the completely different demographic in the study setting relative to the rest of the country. More than three-quarters of the patients with bladder cancer in this study were diagnosed in the early stages of the malignancy (Ta and T1). Fifty percent of participants were histologically diagnosed with more aggressive malignancies (TCC HG and CIS). Detecting bladder cancer by ultrasound and flexible cystoscopy results in expedited, definitive and curative treatment, where possible. Patients with bladder cancer referred within 2 weeks of the onset of their symptoms have been shown to have a $5 \%$ survival advantage over those with more delayed referral. ${ }^{[12]}$ In addition, patients with metastatic disease can be referred to palliative care colleagues almost immediately.

Initially, it can be a challenge to get an OSHC up and running. However, with a dedicated team of nurse practitioners assisting the consultant urologist, the advantages to the patient, the urologist and the hospital are many. Patients require fewer appointments before a diagnosis is made, they can build a relationship with a single urologist, and they can avoid additional anxiety related to a delay in establishing a diagnosis. ${ }^{[13-15]}$ In addition, for hospitals like GSH that service a large rural population, the OSHC is even more relevant. The financial burden of repeated visits can be reduced. One-stop clinic patients are more likely to return for care and to recommend this service, both of which are indicators of patient satisfaction. The hospital can service more patients more effectively and reduce the cost of repeated and often unnecessary visits.

From this retrospective series of haematuria cases, it is worth pointing out that fewer than one-third of all the patients included in the study qualified for referral to GSH for screening based on their geographical status, and the remaining patients should have been cared for at healthcare facilities draining their area (Fig. 5). The biggest drainage area that was serviced at the GSH OSHC was the Cape Flats, where almost half of the patients enrolled should have reported to the closest medical facilities (Fig. 5: Mitchell's Plain District Hospital 37.8\%, Khayelitsha District Hospital $11.5 \%$ ) for screening in the early stages of their symptoms if the service had
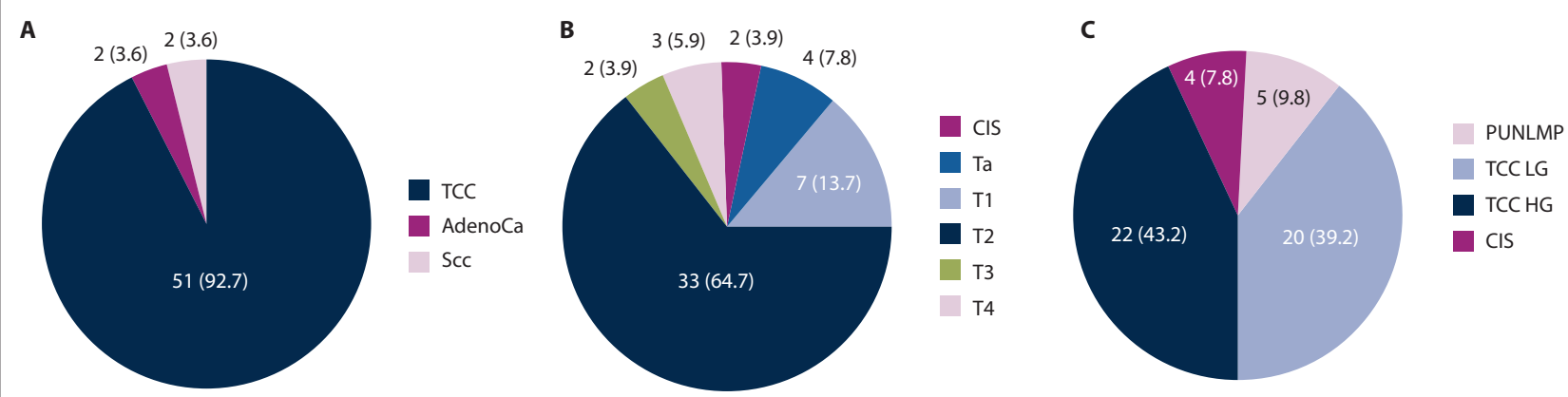

Fig. 4. Type (A), stage (B) and grade (C) of identified tumours. (TCC = transitional cell carcinoma; AdenoCA = adenocarcinoma; SCC = squamous cell carcinoma; CIS = carcinoma in situ; $P U N L M P=$ papillary urothelial neoplasm of low malignant potential; $L G=$ low grade; HG = high grade.) 


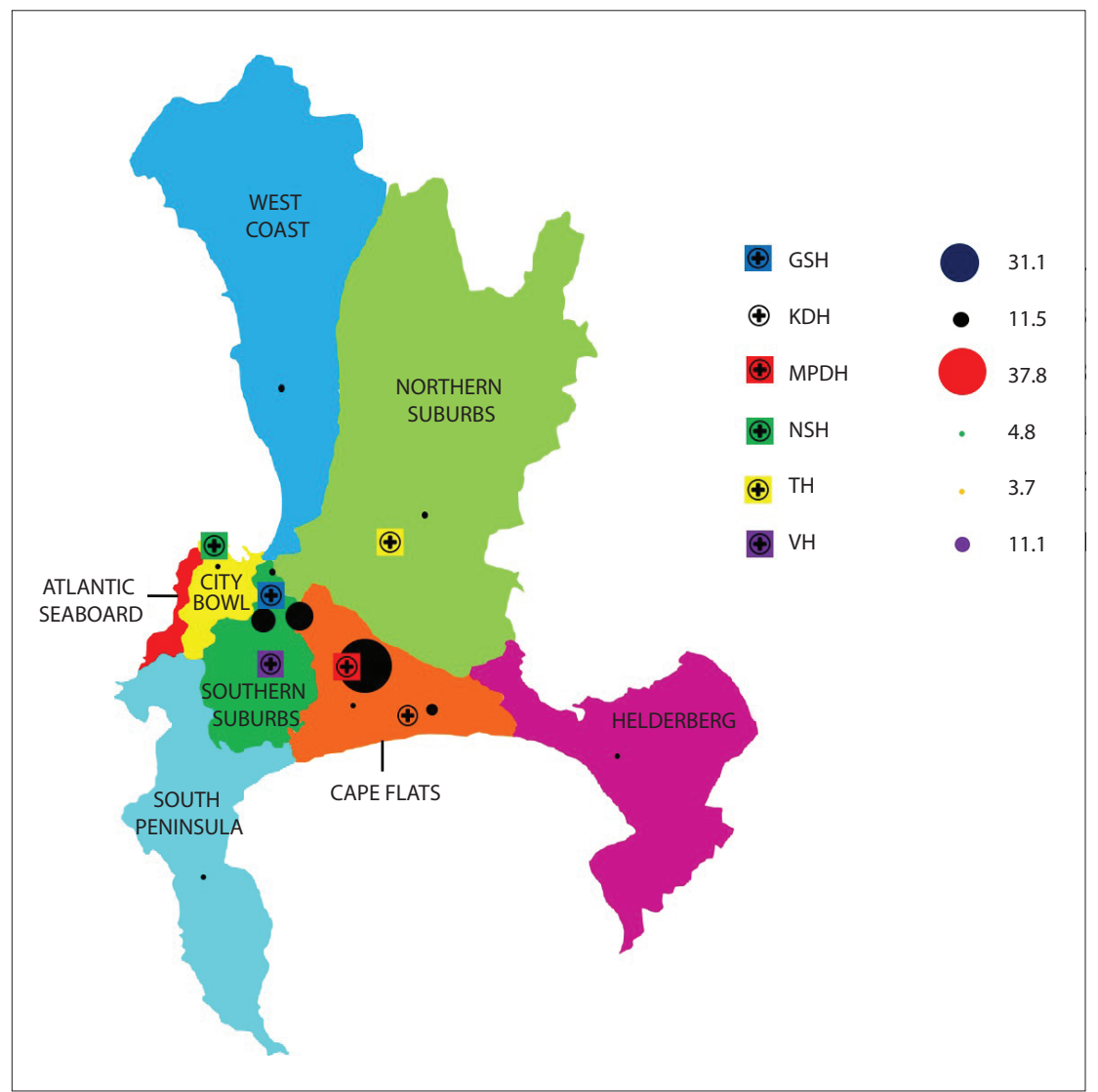

Fig. 5. Geographical coverage of referrals to the GSH one-stop haematuria clinic, with the closed black circles $(\bullet)$ on the map of the Cape Town metropole representing the proportions of patients coming from these respective areas (size determined by proportion). The six secondary and tertiary medical facilities $(G S H=$ Groote Schuur Hospital $; K D H=$ Khayelitsha District Hospital MPDH $=$ Mitchell's Plain District Hospital; $\mathrm{NSH}=$ New Somerset Hospital; $\mathrm{TH}=$ Tygerberg Hospital; $\mathrm{VH}=$ Victoria Hospital) covering the metropole are included in the map to provide a sense of proximity to patients' dwellings. An indication of potential redistribution of referrals to centres closest to patients' dwellings is also provided, with the size of the coloured circles next to the facility names denoting the proportions of referrals that could be redirected to each facility if it was upgraded for haematuria screening.

been available there. Of all cancer cases identified in this study, $80 \%$ could have been screened earlier at their respective healthcare facilities had there been a regular OSHC there, and referred for the appropriate standard of care at a designated tertiary centre.

\section{Conclusions}

The OSHC was designed to be an efficient diagnostic unit where patients undergo tests that confirm the presence or absence of malignancy, thus potentially decreasing periods of delay between diagnosis and treatment. The fact that three-quarters of the cancer diagnoses made in this study were in the early stages is a good reflection of the efficacy of this specialised clinic. It has fast-tracked the management of all patients (both early and late diagnoses), leading to a reduction in morbidity and mortality, and we recommend that similar
Sr Desire Abels, Sr Julia Jacabs, Sr Cecilia Wilson, Mr Phumlani Msi, Mrs Lesley Burke, Mrs Shene Isaacs, Mrs Zubaida Viljoen and Mr Gasant Harris.

Author contributions. SS (first author) and SZJ did the data analysis and wrote up the manuscript, to which they contributed equally. All the clinicians listed as authors were involved in performing the procedures and generating the data published, as well as providing valuable input in the initial phase of drafting the manuscript. JL provided guidance for the draft manuscript.

Funding. None.

Conflicts of interest. None.

1. Mariani A, Mariani M, Macchioni C, Stams U, Hariharan A, Moriera A. The significance of adult hematuria: 1,000 hematuria evaluations including a risk-benefit and cost-effectiveness analysis. J Urol 1989;141(2):350-355. https://doi.org/10.1016/ analysis. J Urol 1989;141

So022-5347(17)40763-4
2. Johnson E, Daignault S, Zhang Y, Lee C. Patterns of hematuria referral to urologists: Does a gender disparity exist? Urology 2008;72(3):498-502. https://doi.org/10.1016/j. urology.2008.01.086

3. Grossfeld G, Litwin M, Wolf J, et al. Evaluation of asymptomatic microscopic hematuria in adults: The American Urological Association best practice policy - part II: Patient evaluation, cytology, voided markers, imaging, cystoscopy, nephrology evaluation, and follow-up. Urology 2001;57(4):604-610. https:// doi.org/10.1016/S0090-4295(01)00920-7

4. Mishriki SF, Grimsley SJS, Nabi G. Incidence of recurrent frank he frank hematuria and urological cancers: Prospective 6.9 years
of followup. J Urol 2009;182(4):1294-1298. https://doi.org/10. of followup. J Urol 2009

5. CANSA Fact Sheet, 2014. https://www.cansa.org.za/files/ 5. CANSA Fact Sheet, 2014. https://www.cansa.org.za/files/
2018/08/Cancer-Registry-2014.pdf (accessed 3 April 2019).

6. Cumberbatch MGK, Jubber I, Black PC, et al. Epidemiology of bladder cancer: A systematic review and contemporary update of risk factors in 2018. Eur Urol 2018;74(6):784-795. https://doi.org/10.1016/j.eururo.2018.09.001

7. Sylvester RJ, van der Meijden APM, Oosterlinck NW, et al. Predicting recurrence and progression in individual patients with stage Ta T1 bladder cancer using EORTC risk tables: A combined analysis of 2596 patients from seven EORTC trials. Eur Urol 2006;49(3):466-477. https://doi.org/10.1016/j. eururo.2005.12.031

8. Edwards TJ, Dickinson AJ, Natale S, Gosling J, McGrath JS. A prospective a of A prospective analysis of the diagnostic yield resulting from the attendance of 4020 patients at a protocol-driven haematuria
clinic. BJU Int 2006;97(2):301-305. https://doi.org/10.1111/ clinic. BJU Int 2006;97

9. Khadra MH, Pickard RS, Charlton M, et al. A prospective analysis of 1,930 patients with hematuria to evaluate current diagnostic practice. J Urol 2000;163(2):524-527. https://doi. org/10.1016/S0022-5347(05)67916-5

10. Turner A, Hendry W, Williams G, Wallace D. A haematuria diagnostic service. BMJ 1977;2(6078):29-31. https://doi.org/10. $1136 / \mathrm{bmj} .2 .6078 .29$

11. Eastern Cancer Registry and Information Centre (ECRIC), One-year stage-specific relative survival rates, adults (ages 15 99), Anglia Cancer Network 2001 - 2005. www.ecric.nhs.uk (accessed 21 September 2017).

12. Carmignani L, Bozzini G, Nicosia V, et al. Hematuria onestop clinic: First experience in Italy with 150 cases. Urologia stop clinic: First experience in Italy with 150 cases. Urolog
2011;78(4):262-266. https://doi.org/10.5301/RU.2011.8873

13. Ooi WL, Lee F, Wallace DM, Hayne D. 'One stop' haematuria clinic in Fremantle Hospital, Western Australia: A report of the
coing clinic in Fremantle Hospital, Western Australia: A report of the
first 500 patients. BJU Int 2011;108(Suppl 2):62-66. https://doi. org/10.1111/j.1464-410X.2011.10711.x

14. Wallace D, Bryan R, Dunn J, Begum G, Bathers S. Delay and survival in bladder cancer. BJU Int 2002;89(9):868-878. https:// doi.org/10.1046/j.1464-410X.2002.02776.X

15. Coull N, Rottenberg G, Rankin S, et al. Assessing the feasibility of a one-stop approach to diagnosis for urological patients. Ann R Coll Surg Engl 2009;91(4):305-309. https://doi.org/10, 1308/003588409X391802

\section{Declaration. None.}

Acknowledgements. This work was made possible through the valuable support of the nursing and administrative staff of the Division of Urology at GSH, especially Sr Millia Sojola,
16. Bateman C. NHI will put GPs 'back at the centre' - Motsoaledi. S Afr Med J 2012;102(12):904. https://doi.org/10.7196/SAMJ. 6475

Accepted 10 April 2019. 